\title{
Factors Influencing the Adoption of Minimally Invasive Surgery
}

Danson Mwaniki Muchiri, George Odhiambo Otieno, Dominic Charles Okero

School of Medicine and Health Sciences, Kenya Methodist University

Correspondence to: Mr. Danson Mwaniki Muchiri, P.O. Box 7731-00100 Nairobi, Kenya. Email: dmwaniki2000@ yahoo.com

\begin{abstract}
Background: Cost is a major concern for delivery of minimally invasive surgical technologies due to the nature of resources required. It is unclear whether factors extrinsic to technology availability impact on this uptake. Objectives: To establish the influence of institutional, patient and surgeon-related factors in the adoption of minimally invasive surgical technologies. Methods: Eighty surgeons in tier 4 hospitals in Nairobi were subjected to questionnaires and key informant interviews between January and May 2015. The respondents were required to cite one surgical procedure for which they had the option of either open or minimally invasive surgical approach (MIS). Of the factors presented, they were required to grade them from 1 for least recurring to 5 for most recurring. Results: The response rate was $100 \%$. A total of 9 surgical interventions emerged from the respondents. The most common impediment to MIS
\end{abstract}

\section{Introduction}

A health system is the sum total of all activities whose primary purpose is to promote, restore and maintain health. The activities are classified into six building blocks namely; service delivery, health workforce, health information, medical technologies/vaccines/ pharmaceuticals, financing and governance (1). The reduction of wastage in the delivery of health services is regarded by the World Health Organization (WHO) as an important aspect of health systems strengthening. Technological advances have led to the development of surgical approaches that minimize wastage and achieve greater outcomes with the available was unavailability of the required equipment and non-functional equipment (mean score, 2.61). The most common patient related factors that led to an open approach were patient presentation, obesity, co-morbidities and age (mean score 2.87). The most common surgeon related factor was lack of confidence in the MIS approach (mean score, 2.55). Conclusions: Absence of enabling functional equipment or device at the time of surgery is the most significant institutional factor that impedes the adoption of MIS. Intraoperative complications and the surgeon's comfort emerged as the most significant patient and surgeon factors respectively.

Keywords: Minimally Invasive Surgical Approaches, Health System Pillars, Service Delivery.

Ann Afr Surg. 2016;13(2): 67-71.

DOI: http://dx.doi.org/10.4314/aas.v13i2.8 resources. One such approach is the adoption of minimally invasive surgery (MIS). Minimally invasive approaches to surgical treatment have been proven to reduce medical spending and absenteeism from work (2) . Reduced absenteeism has a positive net effect on productivity in an economy. Surgical site infections which further lead to high bed occupancies and higher healthcare costs are reduced by MIS approaches $(3,4)$. Clear outlines of reasons for low uptake of these approaches are not available for use by decision makers hence no evidence based remedial actions can be taken as yet. The objectives of this study were to determine the influence of Institutional, Patient 
and Surgeon factors on the utilization of minimally invasive approaches to surgery in tier four hospitals of Nairobi county.

\section{Methods}

This was a cross-sectional descriptive survey carried out among 100 general surgeons and gynecologists working in tier four hospitals in Nairobi county namely the Nairobi, Kenyatta National, Aga Khan, MP Shah and Mater Hospitals. The respondents were recruited from the list using census. The list ensured non-repetitive sampling since some of the surgeons worked in more than one hospital. Data was collected by way of self administered questionnaires and interviews conducted in their clinics at the five hospitals.. The sample size was determined using the Yamane formula (5). Quality control was a continuous process throughout the study to maximize validity and reliability of the findings of the study. A pilot study was carried out on the data collection instrument so as to avoid bias and misinterpretations of the questions as well as for consistency, accuracy and reliability.

The research was approved by the ethics review board of the Kenya Methodist University. The respondents consented to the study, and were informed that it their participation was voluntary.

The quantitative data collected were coded, processed and cleaned of inconsistencies and outliers. The qualitative data were analyzed through the selection of concepts, categories and themes. It involved reading through the data and developing codes that draw similar connections between categories and themes. Quantitative data was analyzed by the use of SPSS version 23 as per the specific research questions using frequencies and percentages. Findings were presented in the form of text, graphs and tables.

\section{Results}

The response rate was $100 \%$. Of the respondents, 41(51.2\%) interviewed were obstetrics/gynecologists while $39(48.8 \%)$ were general surgeons. The respondents cited nine procedures that the option of MIS in addition to open method (Figure 1). Factors influencing choice of open procedures over MIS are summarized in Table 1. The main reasons for opting for open procedures were patient factors such as obesity or preexisting comorbidities, and cost of surgery. Patients who had MIS had a shorter duration of hospitalization compared to their counterparts who underwent open procedures (Table 2).

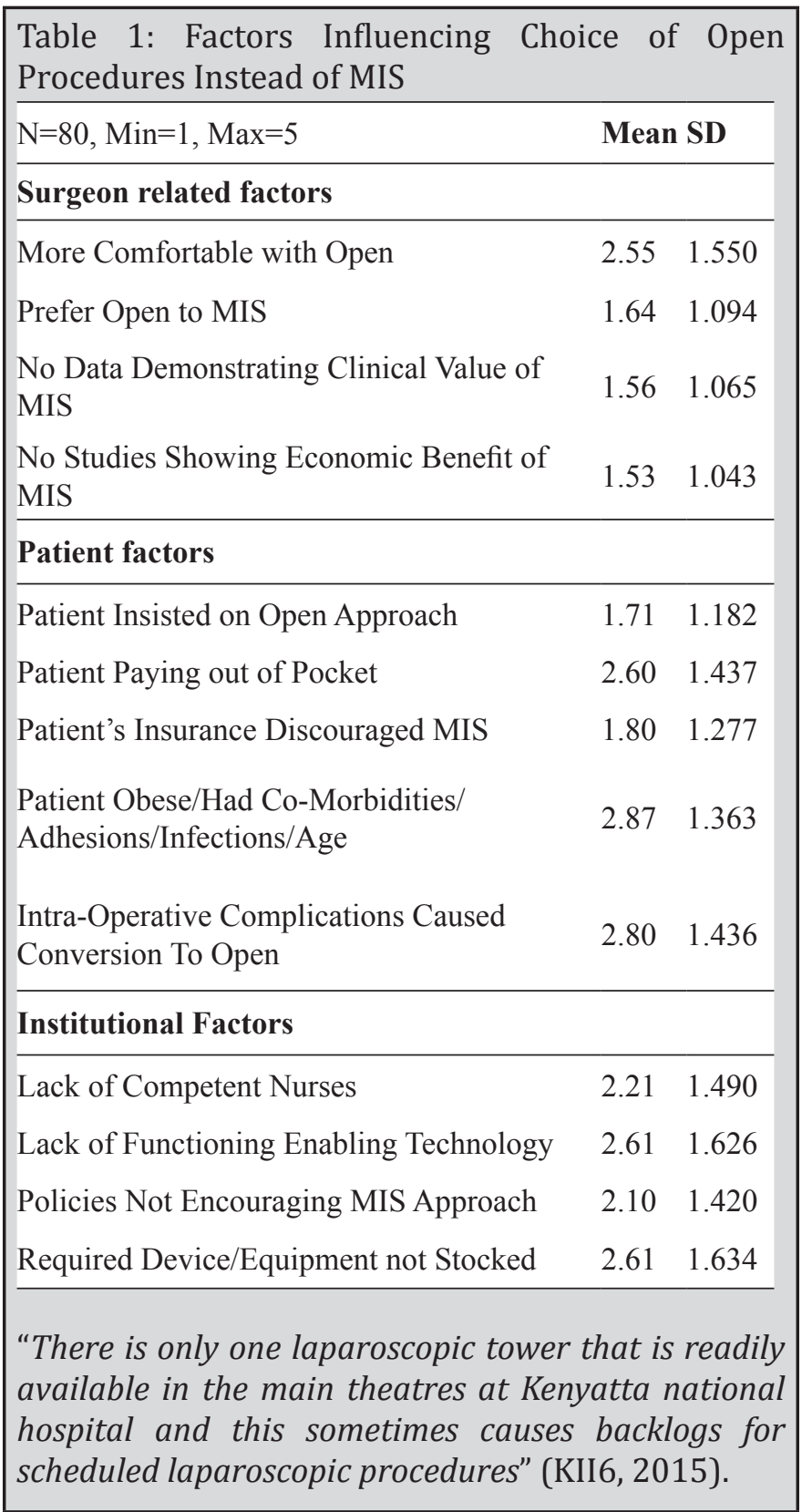

\begin{tabular}{|}
\hline \begin{tabular}{|c|cc|cc|}
\hline Table 2: Average Length of Hospitalization \\
\hline & \multicolumn{2}{|c|}{ MIS } & \multicolumn{2}{c|}{ Open } \\
\hline Days & Frequency & Percent & Frequency & Percent \\
\hline$<1$ & 14 & 17.6 & 0 & 0 \\
1.0 & 27 & 33.8 & 5 & 6.3 \\
1.5 & 5 & 6.3 & 2 & 2.5 \\
2.0 & 20 & 25.0 & 2 & 2.5 \\
2.5 & 4 & 5.0 & 8 & 10.0 \\
3.0 & 6 & 7.5 & 28 & 35.0 \\
3.5 & 2 & 2.5 & 3 & 3.8 \\
4.0 & 2 & 2.5 & 14 & 17.5 \\
5.0 & 27 & 33.8 & 18 & 22.5 \\
\hline
\end{tabular}
\end{tabular}




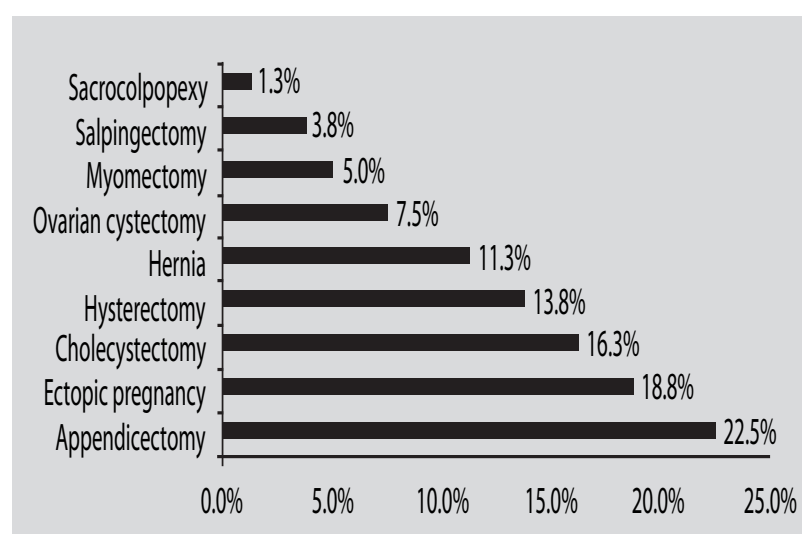

Figure 1: Surgical Interventions Cited

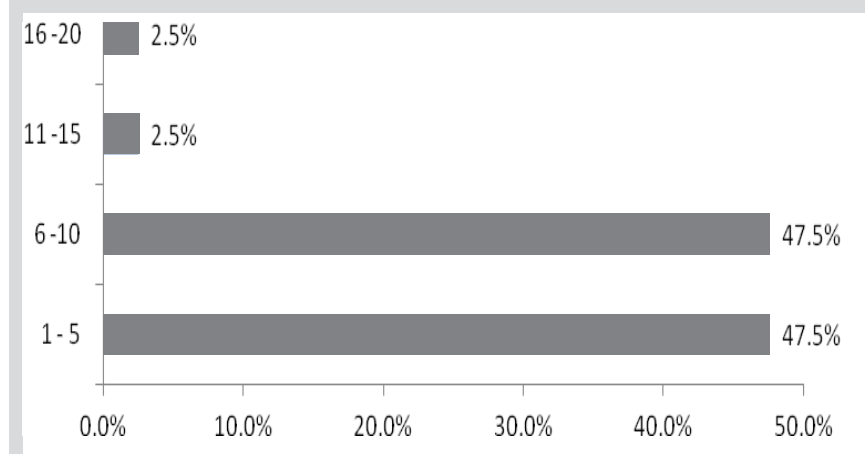

Figure 2: Frequency of Surgeries Done Weekly

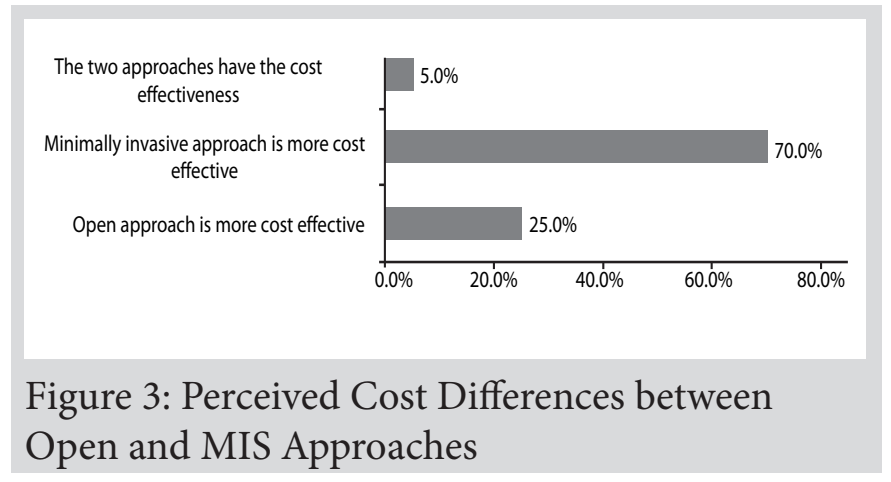

\section{Discussion}

\section{Influence of Institutional Factors}

The study was conducted in tier four hospitals hence the varied responses by the respondents. However, the most common reasons for resorting to open surgical procedures were lack functioning enabling technology and lack of required device/equipment. The respondents ranged from those who exclusively operate in the highly equipped private hospitals to those who operate at the public national referral hospital. High patient numbers and attendant constraints in availability of resources are a reality at the public referral hospital (6). Lack of competent nurses to assist in MIS procedures was the second most common reason why surgeons resorted to the open approach. The high number of operating theatres nursing staff at the national hospital may come with the challenge of wide variations in levels of skill within the staff who may have assisted different respondents at different times. The effect of wide variations in levels of skill across the large numbers of nursing staff is counterbalanced by the high volumes of patients. High volumes of surgical patients improve the level of skill of the involved staff and consequently the outcomes (7). Studies have demonstrated reduction in death rates and median hospital stay in high volume centers when compared to lower volume centers (8). Although a high-surgeon volume correlated with lowered mortality studies have found that highvolume hospitals demonstrated improved outcomes, highlighting the importance of other hospital factors other than surgeon's expertise in determining surgical outcomes (7).

Most respondents did not cite hospital policies as an impediment to the adoption of MIS implying that no hospital studied has in place policies that discourage the adoption of minimally invasive approaches. Subsequent training in laparoscopic surgery outside of the initial training is a predictor of lower rates of complications (9). It can therefore hold that hospital or wider health system policies that encourage continuous education for staff and surgeons is supportive of MIS adoption.

\section{Influence of Patient Factors}

Patient presentation (obesity/co-morbidities/ adhesions/infections/age) was the most recurring reason why surgeons resorted to open approaches. The American College of Obstetricians and Gynecologists recommended a vaginal hysterectomy for the obese patient citing better outcomes and lesser risks of complications than for either laparoscopic or abdominal approaches (10). Pre-existing conditions in the patient have been documented to influence the decision on the surgical approach in cholecystectomy (11). Intra-operative complications were the second most common cause of conversion to open surgery. Reasons for conversion to open have been studied previously in cholecystectomy and include inability to correctly identify anatomy, intra-operative bleeding, suspected choledocholithiasis, and suspected bile duct injury (12). Paying out of pocket was ranked third in importance. Most patients in Kenya make out of pocket payments for health services (13). A study in the US reported that $78 \%$ of the physicians took into consideration the out of pocket costs to the patient when making prescribing decisions, considering care setting, and when considering diagnostic tests (14) . In health systems where most patients pay for their own care, a patient's socioeconomic status has been found to influence clinical management decisions to suit the patient's financial situations (15). The least significant 
factor amongst the considered factors was patient's request for an open approach. Patients' wishes have been shown to influence doctors' decisions on care, including admission to an intensive care unit (15).

\section{Durations of Hospitalization}

This study's findings of reduced hospital stay in MIS procedures are corroborated by the findings of a prospective case series conducted at the Aga Khan Nairobi (11). Surgical approach whether open or minimally invasive has a bearing on lengths of hospitalization and hence overall costs of treatment (16). Furthermore, MIS approaches have been associated with the conservation of patient's own blood translating to lower rates of transfusion which in turn shorten the durations of hospitalization (17).

\section{Surgeon Related Factors}

The implications of these results for the influence of surgeon related factors indicate that, level of surgeon's comfort with a certain surgical approach is the most important determinant of whether that particular approach will be adopted in a surgical procedure. The WHO listed user error amongst the top three factors that contribute to device related adverse events in patients (18). A study of 2000 adverse events in Australia involving devices showed that only $9 \%$ were purely a result of equipment failure, the vast majority being a result of improper use (19). A study on the positive predictors of successful surgical outcome in laparoscopic hysterectomy showed that level of surgeon experience positively predicts a favorable outcome for the patient, with an improvement apparent after each surgery for at least the first 125 surgeries (20). Gaps in basic laparoscopic suturing skills were repeatedly cited as an impediment to the adoption of minimally invasive approaches. The incorporation of MIS training during residency leads to a higher prevalence of MIS approaches. The Aga Khan hospital's inclusion of MIS approaches to residency training might be attributed to its position as the pioneer of laparoscopic surgery in Nairobi. A retrospective study of laparoscopic surgeries done in Nairobi between 2000 and 2002 showed that $85.35 \%$ were performed at the Aga Khan (21).

Although preference for open procedures ranked second after level of comfort as a reason why the respondents would resort to the open approach, it was still an important factor. A study that was done on the preferences of endoscopic surgeons in hernia surgery concluded that surgeons who take the minimally invasive approach repair as the first option for inguinal hernias are convinced that not all hernias are good options for this approach (22). Lack of clinical data on the clinical value of MIS ranked third.
This can be attributed to the fact that existing clinical findings weigh heavily in favor of minimal disruption of tissues during surgery. Respondents cited the lesser risks of adhesion formation postoperatively after MIS compared to open. Furthermore, MIS approaches are associated with superior postoperative cosmetic results (23).

\section{Perceptions on Cost Differences}

Majority of the respondents thought that MIS was more cost effective for the patient. Studies have shown that a physician's perception of costs can influence the clinical decisions taken. However the extent of consideration for the implication of decisions on the out of pocket costs seemed to decrease as the complexity of diagnosis and treatment options increased (14). These findings concur with other studies that have been done on cost differences for the two approaches demonstrating reduced costs in MIS. Researchers from the University of Pennsylvania assessed six types of surgery associated MIS approaches with lower work absenteeism costs due to the associated quicker recoveries (2). The adoption of MIS approaches in a hospital involves the acquisition of new technologies. The element of cost therefore cannot be downplayed. The role of device manufacturers in the uptake of new technologies has been outlined by experts in the industry. It includes the development of reimbursement strategies for purposes of insurance where applicable and this incorporates economic evaluation (24). Evaluation for devices faces challenges since the guidelines have been written with pharmaceutical products in mind (25). The evaluation has to factor in user characteristics and the learning curve (26). This implies that it would be of benefit to the manufacturers to shorten the learning curve for a new device by investing in training for the user for a more efficient evaluation process.

\section{Conclusions}

Absence of enabling functional equipment or device at the time of surgery is the most significant institutional factor that impedes the adoption of MIS. Unfavorable patient presentation such as obesity, adhesions and other co-morbidities was the most significant patient impediment. Surgeon's level of comfort with an approach/enabling technologies was the most significant factor amongst the considered surgeon related factors.

The gynecologists and general surgeons who participated in the study. 


\section{References}

1. World Health Organization, Everybody's Business: Strengthening Health Systems to Improve Health Outcomes: WHO's Framework For Action. Geneva, Switzerland: World Health Organization, 2007.

2. Epstein AJ, Groeneveld PW, Harhay MO, et al. Impact of Minimally Invasive Surgery on Medical Spending and Employee Absenteeism. JAMA Surg. 2013;148(7): 641-7

3. Ee WWG, Lau WLJ, Yeo W, et al. Does Minimally Invasive Surgery Have a Lower Risk of Surgical Site Infections Compared With Open Spinal Surgery? Clin Orthop Relat Res. 2014; 472(6):1718-24.

4. Pasam RT, Esemuede IO, Lee-Kong SA, et al. The Minimally Invasive Approach is Associated with Reduced Surgical Site Infections in Obese Patients Undergoing Proctectomy. Techniques in Coloproctology. 2015:19(12):733-43.

5. Taro Yamane, Statistics: An Introductory Analysis, 2nd ed. New York: Harper and Row, 1967.

6. Auditor General, "Performance Audit Report of the Auditor - General: Specialized Healthcare Delivery at Kenyatta National Hospital Waiting - time for Cancer, Renal and Heart Patients," Nairobi, 2012.

7. Torrente JE, Cooney RN, Rogers AM, et al. Importance of Hospital Versus Surgeon Volume in Predicting Outcomes for Gastric Bypass Procedures. Surg Obes Relat Dis. 2013; 9(2): 247-52.

8. Gouma DJ, van Geenen RCI, van Gulik TM, et al. Rates of Complications and Death After Pancreaticoduodenectomy: Risk Factors and the Impact of Hospital Volume. Ann Surg. 2000;232(6):786-95.

9. See WA, Cooper CS, Fisher RJ. Predictors of Laparoscopic Complications After Formal +Training in Laparoscopic Surgery. JAMA. 1993; 270(22): 2689-92.

10. American College of Obstetricians and Gynecologists, "Gynecologic surgery in the Obese woman.," Washington, DC, 2015.

11. Patel SC, Bhatt JR. Laparoscopic Cholecystectomy at the Aga Khan Hospital, Nairobi. East Afr Med J. 2000; 77(4):194-8.

12. Bingener-Casey J, Richards ML, Strodel WE, et al. Reasons for Conversion from Laparoscopic to Open Cholecystectomy: A 10-Year Review. J Gastrointest Surg. 2002; 6(6): 800--5.

13. Chuma J, Okungu V. Viewing the Kenyan Health System Through an Equity Lens: Implications for Universal Coverage. Int J Equity Health. 2011;10(22): 1-14.
14. Pham HH, Alexander GC, O’Malley AS. Physician Consideration of Patients' Out-of-Pocket Costs in Making Common Clinical Decisions. JAMA Intern Med. 2007; 167(7): 663-8.

15. Hajjaj FM, Salek MS, Basra MKA, et al. NonClinical Influences on Clinical Decision-Making: A Major Challenge to Evidence-Based Practice. J R Soc Med. 2010; 103(5); 178-187.

16. Lucio JC, VanConia RB, DeLuzio KJ. Economics of Less Invasive Spinal Surgery: An Analysis of Hospital Cost Differences Between Open and Minimally Invasive Instrumented Spinal Fusion Procedures During the Perioperative Period. Risk Manag Healthc Policy. 2012; 5: 65-74.

17. Weber EW, Slappendel R, Prins $M H$, et al. Perioperative Blood Transfusions and Delayed Wound Healing After Hip Replacement Surgery: Effects on Duration of Hospitalization," Anesth Analg. 2005; 100(5): 1416-21.

18. World Health Organization, "Medical devices: Managing the Mis-march; Increasing complexity of medical technology and consequences for training and outcome of care," Geneva, Switzerland, Background Paper 42010.

19. Fouladinejad F, Roberts JR. An Analysis of Training Activities in the Use of Critical Care Equipment Within Hospitals in the United Kingdom. Anaesth. 1998; 53(8); 810-7.

20. Twijnstra AR, Blikkendaal MD, van Zwet EW. Predictors of Successful Surgical Outcome in Laparoscopic Hysterectomy. Obstet Gynecol. 2012; 119(4): 700-8.

21. Parkar RB, Thagana NG, Baraza R, etal. Experience with Laparoscopic Surgery at the Aga Khan Hospital, Nairobi. East Afr Med J. 2003; 80(1): 44-50.

22. Morales-Conde S, Socas $M$, Fingerhut A. Endoscopic Surgeons' Preferences for Inguinal Hernia Repair: TEP, TAPP, or OPEN. Surg Endosc. 2012; 26(9): 2639-43.

23. Dutta S. Early Experience with Single Incision Laparoscopic Surgery: Eliminating The Scar from Abdominal Operations. J Pediatr Surg. 2009; 44(9): 1741-45.

24. Nancy JStark and Vincent Jaeger, "Reimbursement Strategies for New Technologies," Chicago, IL, CDG Whitepapers 2011.

25. Tarn TY, Smith MD. Pharmacoeconomic Guidelines Around the World. ISPOR CONNECTIONS. 2004; 10: 4-5.

26. Sorenson C, Tarricone R, Siebert M, et al. Applying Health Economics for Policy Decision Making: Do Devices Differ from Drugs? Eurospace. 2011; 13(2): ii54-8. 